\title{
Research on Tourism E-commerce based on Data Mining
}

\author{
Yan $\mathrm{Liu}^{1,2, a}$ \\ ${ }^{1}$ School of Economics and Management, Southwest JiaoTong University, China \\ ${ }^{2}$ College of Tourism, Sichuan Agricultural University, China \\ ayanliu0422@sina.com
}

\begin{abstract}
This paper describes in detail the web data mining technology, analyzes the relationship between the data on the web site to the tourism electronic commerce (including the server log, tourism commodity database, user database, the shopping cart), access to relevant user preference information for tourism commodity. Based on these models, the paper presents recommended strategies for the site registered users, and has had the corresponding formulas for calculating the current user of certain items recommended values and the corresponding recommendation algorithm, and the system can get a recommendation for user.
\end{abstract}

Keywords-data mining; tourism e-commerce; web data mining; recommended system.

\section{INTRODUCTION}

Tourism electronic commerce development main have portal site, professional tourism website, the traditional tourism business travel website at present. The content of these sites is mostly simple introduction of enterprises, the main tourist routes, attractions, go out to try at home and travel works etc.. The technology is only static or dynamic not static website Webpage custom style, no way to achieve rapid update framework. There are also problems in system porting, cannot cross platform, scalability is poor, no component construction thought. But China's tourism electronic commerce transactions are still in the traditional mode: (1) the hotel reservations in the phone book, the payment; (2) to telephone booking, ticket booking door payment; line booking by telephone booking, payment etc.. ${ }^{[2]}$; (3) travel website should establish tourism information perfect system, and component extension, can be updated framework, technology should be platform for developing multi-tier architecture model.

In many of these tourism e-commerce and frequent trading, people have made quite a lot of data, this to other people or business institutions researchers numerous potential opportunities to gain insight into the user experience, business marketing, personal preferences and usually so-called human behavior ${ }^{[1]}$. In this paper the method of using Web data mining, can design a recommended offer for tourism e-commerce system.

\section{ARCHITECTURE OF TOURISM E-COMMERCE PLATFORM BASED ON INFORMATION RELEASE SYSTEM}

Tourism e-commerce or e-government framework (Fig.1) contains the presentation layer, application layer and application support layer, system software support layer, physical layer network layer. Based on the general framework of e-commerce platform information release system (Fig.2) belongs to the e-commerce or e-government framework of the application layer and the application support layer, that can build up in the system software support layer of this framework, provides the application layer and the component services application support layer to layer.

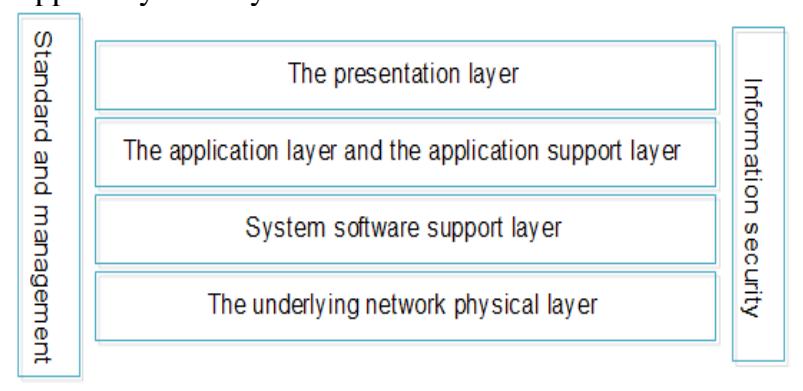

Figure 1. The overall architecture of e-commerce platform

The platform is divided into 2 levels: (1) electronic commerce platform on the information release system; (2) information publishing system based on the characteristics of electronic commerce website. Based on the platform of electronic business information publishing system, including platform supporting collaboration system, platform customized website mode code database (data layer, on the platform outside the system belongs to the platform but part of a system), system, e-commerce platform for information construction of e-commerce platform release system, platform template parsing system and platform system.

Based on the information system of the characteristics of the electronic commerce site features, including hotel reservations, car rental, meeting scheduled assembly components, assembly line scheduled predetermined components and ticket booking module etc.. These components formed the e-commerce portal on real significance present for customer. User interaction with the platform of business process is divided into the following two categories:

(1) Process of electronic commerce platform

Platform to build website construction platform framework through system memory from the model code and call mode code, also call platform supporting collaboration system, complete with their business outside support function. In addition, platform information system 
according to the model building system requirements part code loading platform, construct information publishing framework, also call platform supporting collaboration system, complete with their business outside support function.

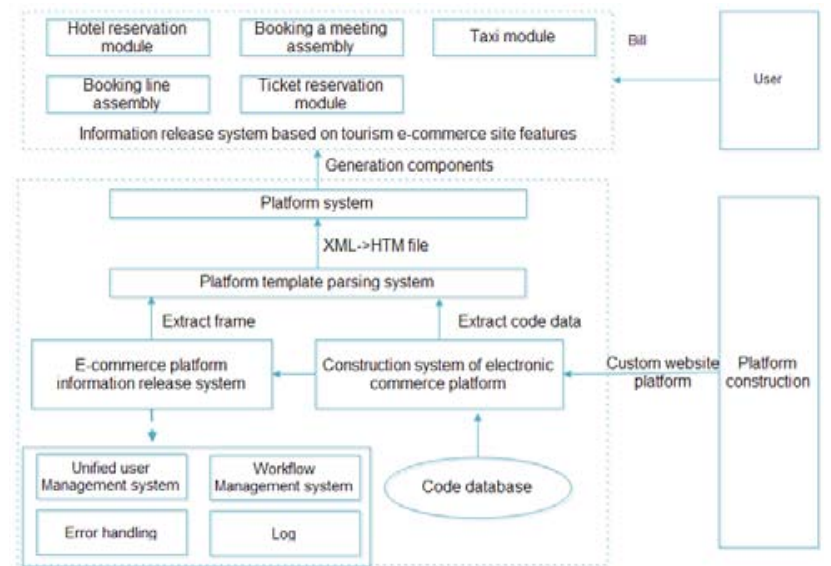

Figure 2. The general framework of e-commerce platform information release system

Platform template parsing system release system and platform from the platform construction of information system are extracted from the filled XML data tree generated framework and model code data, and filled with data XML file for conversion of XSLT template parsing, finally generate the HTML file. Platform system extraction to generate the HTML file from the platform template parsing system are combined, show portal components to generate custom.

(2) The user initiated process

Website login users fill out web forms for information exchange, information from the site into the system, after treatment with the underlying data layer interaction, and then along the opposite direction results, show the user finally.

\section{RECOMMENDED STRATEGIES FOR TOURISM E- COMMERCE}

\section{(1) Items similarity}

Item similarity refers to the universal existing relationship of the item $i$ and item $j$ when customer buy it, with $\operatorname{sim}(i, j)$ expression. Corresponding to the actual business operation, $\operatorname{sim}(i, j)$ said to buy things on the site of the user, if they buy the goods " $i$ " will usually buy goods "probability. In other words, $\operatorname{sim}(i, j)$ represent probability of $i$ and $j$ items is purchased together. The greater the probability, we say that the two items more similar. This model can be used to guide the implementation of cross selling task recommendation system.

Calculate similarity based on cosine: using this method, we put the two item $i$ and $j$ as two dimensional vector $m$ user space. The computation of similarity is calculated the angle between the vectors representing these two items of cosine. If the user " $u$ " purchased items $i$, then matrix in section $u$ for the " $i$ " column value is 1 , otherwise 0 . In general, $\mathrm{i}$ and $\mathrm{j}$ similarity calculation formula for goods is:

$$
\operatorname{sim}(i, j)=\cos (\vec{i}, \vec{j})=\frac{\vec{i} \bullet \vec{j}}{|\vec{i}| \times|\vec{j}|}
$$

$\vec{i} \bullet \vec{j}$ Indicates the number of product of vector, $|\vec{i}|$ and $|\vec{j}|$ is respectively norm of vector.

(2) The user clustering model

According to the user visit on the site to generate clustering, similar access behavior of users will be clustered into a class. It is worth noting that for the registered users, each registered users themselves to form a category.

Transaction clustering, using ${ }^{[3]}$ the most widely used $\mathrm{K}$-means algorithm in data mining. Before using the clustering algorithm K-means transaction, the transaction must be expressed as a numeric vector K-means to operation. In order to vector to conduct the affairs of representation, we can put the transaction mapping in the page space, a transaction is expressed as a multi-dimensional vector space. Set up site all web pages for $\mathrm{P}=\left\{\mathrm{p}_{1}, \mathrm{p}_{2}, \ldots \mathrm{p}_{\mathrm{n}}\right\}$, the transaction $\mathrm{t} \in \mathrm{T}$ can be expressed $t=<w\left(P_{1}, t\right), w\left(P_{2}, t\right), \ldots, w\left(P_{n}, t\right)$ in multi dimension vector page space $\mathrm{P}$, which:

$$
w\left(p_{j}, t\right)=\left\{\begin{array}{c}
\gamma \text { number of page } p_{i} \text { that appearinaffairt } \\
0 \text { number of page } p_{i} \text { that don't appearinaffairt } 1 \leq j \leq n, \gamma>0
\end{array}\right.
$$

Identified transactions are transformed into a multidimensional vector on the space pages $P$, the uid is the clustering algorithm based on K-means for empty the affairs.

\section{(3) Establish items - Page Mode}

We need to analysis and identify the page of the goods involved information extraction methods of data mining. Information extraction methods on the site on the HTML or XML page uses information extraction, extract the relevant items of information from HTML or XML, you can use $[2,20]$ method, the use of machine learning theory to the items of information extraction.

The information extraction of HTML and XML can obtain the weights $\mathrm{W}$ (I, P) that relative to this page or each page relates to the items set and these items, for example, the weight can be defined as frequency of the goods $\mathrm{i}$ appear on the pages $\mathrm{P}$. Reset all items web site involves the composition space for the Is (Item Space), the space size is $n$. So in the information extraction, each page can be represented as an n-dimensional vector objects in $\mathrm{P}$ space, establishing item - Page Mode $p=\left\{w_{1}^{p}, w_{2}^{p}, \ldots, w_{n}^{p}\right\}$. Among them:

$$
w_{i}^{p}=\left\{\begin{array}{c}
w(i, p) \text { weight of goodsi in page } p \\
0 \text { if page pthat don't ralated to goodsi }
\end{array} \quad 1 \leq i \leq n\right.
$$


(4) The establishment of user clustering model about items

Through the web usage mining produces a user clustering similar access behavior, information extraction through web content mining produced a page in the object space Is vector representation. Using them as the basis, can produce goods - user clustering model. Item - user mode is a kind of user to goods space Is on an item's interest, denoted as: $\varphi(\mathrm{i}, \mathrm{c})$ or $\varphi(\mathrm{i}, \mathrm{u})$. The formula for items - user clustering model:

$$
\begin{aligned}
& \varphi(i, c)=\frac{1}{|c|} \sum_{i=1, p \in c}^{n} w(i, p) \times \text { weight }(p, c) \\
& \varphi(i, u)=\frac{1}{|u|} \sum_{i=1, p \in u}^{n} w(i, p) \times w e i g h t(p, u)
\end{aligned}
$$

Where $w(i, p)$ is weight of items i relative to the page $p$, weight $(p, e)$ or weight $(p, u)$ is $p$ relative to c or u page clustering weights. $\mathrm{N}$ is a space the size Is of the items. Then for every cluster $\mathrm{c}$ or $\mathrm{u}$ can be expressed with respect to the n-dimensional vector the clustering weights for space objects, user clustering model items:

$$
\begin{gathered}
c=<\varphi(1, c), \varphi(2, c), \ldots, \varphi(n, c)>\text { or } \\
u=<\varphi(1, u), \varphi(2, u), \ldots, \varphi(n, u)>
\end{gathered}
$$

(5) Recommendation strategy

For the recommendation of the registered users, we mainly consider 4 main factors from strategy.

1) Evaluation value Ru of item i that ever buy by user;

2) Correlation similarity Sim(i,j) between users have bought items i and recommended values are calculated items ;

3) The date and time DT (i) of item i that was choose and buy by the user;

4) Item-user clustering mode $\varphi(\mathrm{j}, \mathrm{u})$ of Items $\mathrm{j}$ that the recommended values are calculated when the user access to the website long time ago;

The calculation formula of goods $\mathrm{j}$ that the goods whether goods $\mathrm{j}$ can be recommended to the user $\mathrm{u}$ are as follows:

$$
\operatorname{Re} c_{u}(j)=\varphi(j, u)+\sum_{i \in u} \operatorname{sim}(i, j) *\left(R_{u}+1\right) * T(i)
$$

"i" is a purchased items of user u during a period of registration in the database, or is the items in the shopping cart.

Parameter $R_{u}$ is the evaluation value that the user have bought items. Here use $R_{u}+1$ as a multiplication factor, in order to avoid is equal to 0 , the items in the formula in the other involved in the calculation of the value of failure.

$\operatorname{Sim}(\mathrm{i}, \mathrm{j})$ is associated similarity that calculated articles $i$ and $j$ earlier. Used as a factor in this formula, said that if the correlation similarity of user u that have high values for purchased item $\mathrm{i}$ and item $\mathrm{j}$, it has also a great possibility that users buy goods $i$ and $j$. So sim (i,j) affect the calculation recommended value items $\mathrm{j}$ in the very great degree.

Parameter $\varphi(\mathrm{j}, \mathrm{u})$ is a reflection of the user u used to visit the item $\mathrm{j}$. Here we consider the user $\mathrm{u}$ has high value access to the goods $\mathrm{j}$, the more he is interested in the good j.

$\mathrm{T}$ (I) is the item I purchase date time and the computation time of a function of two variables: $T$ (I) $=f$ (DT(), DT(i)). If the purchase date and time for good i from the current date is long, so it can produce recommended value is small, or more. So T (I) in the formula is a decreasing function of a mathematics.

\section{THE OPERATING STEPS OF RECOMMENDATION ENGINE}

System find similarity relation according to the relationship between the tourism goods and articles involved they request the latest page, find can recommend items. If an item in the current user requests the page weight high, so high and the articles of association of the similar items can be used as recommended object.

When users browse the site, the session manager (SM) responsible for communicate with the user, to capture the user at the same time related information and send it to the dialogue manager (DM), to call in maintaining and processing context and user interaction and recommender (RE), in the recommenders to complete the calculation recommend the result set, the set of session manager information, the sender shall submit the results to the Web server, complete the recommendation work. Among them, the context of user interaction with the system is recorded in the dialogue context.

\section{CONCLUSIONS}

As we realize the separation of offline part and online part, which can adapt to the large amount of data, greatly improve the scalability of personalization recommendation service and real-time response speed. At the same time, the integration of Web mining technology, in the use of data is relatively small, or Web site contents change frequently, also can provide high quality and personalized recommendation service. Through the interaction of the two module, this system can guarantee the finally presented to customers recommend collection in real time, the new, it is reasonable.

\section{REFERENCES}

[1] Wang zhong. The strategic value and inspiration to promote technology development of large data America[J]. Chinese development was observed in 2012 (6): 44-45.

[2] Lu Nan, Zhou C.G. research on web data mining heterogeneous data integration problem [J].Journal of Shenzhen University (SCIENCE EDITION), 2002, (9).

[3] Alex Berson. Construction of the CRM data mining application [M]. Beijing: People's post and Telecommunications Press, 2001

[4] Song Q.B, Shen Junyi. Web log mining algorithm of multi energy efficient [J]. "Research and development" computer in 2001 third.

[5] Wang Jicheng, Pan Jingui, Zhang Fuyan. Research on Web text mining technology [J]. 2000.5. 